DOI: 10.1007/s00350-008-2282-9

\section{Het recht op de dood. Een juridische beschouwing over mensen die ,lijden aan het leven" en het (ontbrekende) recht op hulp bij zelfdoding onder de huidige Euthanasiewet.}

Coleta van Swal, Celsus juridische uitgeverij, Tilburg (Niederlande) 2008, $116 \mathrm{~S}$., kart., $€ 30,70$

Die Diskussion um die durch das Spannungsverhältnis von Patientenautonomie und (strafrechtlichem) Lebensschutz aufgeworfenen Fragen ist auch in Deutschland von ungeminderter Aktualität. Dies lässt es gerechtfertigt erscheinen, den Blick auf diejenigen ausländischen Rechtsordnungen zu richten, die - wie die niederländische - das Problemfeld der Sterbehilfe einer eingehenden gesetzlichen Regelung zugeführt haben. Die hier zu besprechende, in niederländischer Sprache vorliegende Publikation widmet sich der in den Niederlanden viel diskutierten Frage, ob die durch das Euthanasiegesetz vorgesehene Straffreistellung der Tötung auf Verlangen bzw. der Beihilfe zum Selbstmord durch einen Arzt (Artt. 293 II, 294 II 2 nlStGB) auch dann eintritt, wenn sich das dem Ersuchen des Patienten zugrunde liegende Leiden nicht aus einer (gravierenden) somatischen oder psychischen Erkrankung, sondern maßgeblich aus dem mit fortgeschrittenem Alter zunehmenden Lebensüberdruss des Betroffenen oder einem Gefühl des Abgeschlossenhabens mit dem Leben speist.

Im ersten Kapitel (S. 5 ff.) zeichnet die Verf. den grundlegenden Konflikt zwischen der ärztlichen Pflicht zum Lebensschutz und der Pflicht zur Achtung des individuellen Selbstbestimmungsrechts des Patienten sowie zur Abwendung unnötigen Leids am Ende des Lebens nach, der sich für die von ihr erörterte Problematik als bestimmend erweist. Weiterhin skizziert die Verf. die Entwicklung der für die strafrechtliche Beurteilung lebensbeendenden Handelns maßgeblichen Sorgfaltsanforderungen in der Rechtsprechung der Strafgerichte, aus der hier vor allem die Entscheidung des Hohen Rates im Fall des Hausarztes Sutorius (2002) hervorzuheben ist. Sutorius hatte seinem 86jährigen Patienten Brongersma, der unter einigen Altersbeschwerden und zunehmender sozialer Isolation, nicht hingegen an einer schweren oder gar irreversibel tödlich verlaufenden Krankheit litt, Beihilfe zum Selbstmord geleistet. Der Hohe Rat urteilte, bloßer Lebensüberdruss sei kein tauglicher Grund für die Rechtfertigung lebensbeendender Maßnahmen; vielmehr müsse dem Todeswunsch eine medizinisch klassifizierbare somatische oder psychische Krankheit zugrunde liegen, da nur die Beurteilung derartiger Sachverhalte in die Zuständigkeit des Mediziners falle.

Die Wiedergabe der durch die juristische Aufarbeitung des Falles Brongersma/Sutorius ausgelösten gesellschaftlichen und fachdisziplinären Diskussion ist Gegenstand der Kapitel 2 und 3 (S. $21 \mathrm{ff} ., 49 \mathrm{ff}$.) Eine ausführlichere Würdigung erfährt der im Auftrag der niederländischen Ärzteorganisation KNMG erstellte Bericht der DijkhuisKommission, der die Trennschärfe des durch den Hohen Rat aufgestellten Krankheitskriteriums in Zweifel zieht und eine ärztliche Zuständigkeit in Fällen existentieller Perspektivlosigkeit nicht von vornherein für ausgeschlossen hält. Noch vor Ergehen der Entscheidung des Hohen Rats hatte demgegenüber die Regierung im Gesetzgebungsverfahren zum Euthanasiegesetz eine Einbeziehung bloßen „Leidens am Leben“ in den Anwendungsbereich des Gesetzes ausdrücklich abgelehnt.

Die Kapitel 4 und 5 (S. 65 ff., 95 ff.) dienen der Gegenüberstellung der für und gegen eine Subsumtion der beschriebenen Fallgestaltungen unter das Euthanasiegesetz vorgebrachten Argumente und der Erarbeitung einer eigenen Position durch die Verf. Während Gegner einer Erstreckung der gesetzlichen Regelung auf Fälle bloßen Lebensüberdrusses neben einer restriktiven Konturierung des ärztlichen Kompetenzbereichs häufig religiöse Erwägungen sowie die Gefahr einer „Aushöhlung“ des (Fremd-)Tötungstabus anführen, argumentieren Befürworter vor allem mit dem Autonomiegedanken. Insoweit weist die Verf. allerdings zutreffend darauf hin, dass durch die Einbeziehung des Arztes in das Tötungsgeschehen ein gesteigerter Sozialbezug und damit ein zusätzlicher, durch die Referenz auf das Selbstbestimmungsrecht nicht ohne weiteres auszugleichender
Rechtfertigungsbedarf entsteht. Als überzeugender erweist sich der Hinweis auf den Wortlaut des Gesetzes, welches die Straflosigkeit vom Vorliegen eines als aussichtslos und unerträglich empfundenen Leidens abhängig macht, über dessen Quellen jedoch keine Aussage trifft. Hinzu kommt die Feststellung, dass sich medizinische Klassifikationssysteme zwar als hilfreich bei der Diagnosestellung erweisen mögen, für eine exakte Bestimmung der Grenzen strafbaren Verhaltens jedoch nur unzureichend geeignet sind. In der Etablierung des Krankheitskriteriums durch den Hohen Rat wird daher eine Gefahr für die Rechtssicherheit bei Ärzten und Patienten gesehen, deren Stärkung ein wichtiges Ziel des Gesetzgebers bei der Schaffung des Euthanasiegesetzes war.

Es überrascht nicht, dass sich die Verf. im Ergebnis für eine Einbeziehung der hier behandelten Fallgruppe in den Anwendungsbereich des geltenden Euthanasiegesetzes ausspricht. Man mag dies mit dem Hinweis auf die ausdrücklichen, einer derartigen Ausweitung entgegenstehenden Äußerungen der Regierung im Gesetzgebungsverfahren kritisieren; durch die Verwendung offener, ausfüllungsbedürftiger Begriffe bei der Normierung der zur Rechtfertigung führenden Sorgfaltsanforderungen ist eine eindeutige Verankerung dieser Position im Gesetzestext allerdings unterblieben. Von der Beurteilung der in Rede stehenden Verhaltensweisen nach geltendem niederländischem Recht ist die Frage zu trennen, ob ihre Straffreistellung überhaupt wünschenswert erscheint. Dass die Suche nach einer gesellschaftlich konsensfähigen Antwort auf diese Frage von unverminderter Dringlichkeit ist, macht eine neuere Evaluationsstudie zur Rechtswirklichkeit des Euthanasiegesetzes (Evaluatie Wet toetsing levensbeeindiging op verzoek en hulp bij zelfdoding, Den Haag 2007, www.zonmw.nl) deutlich, aus der hervorgeht, dass im Jahr 2005 etwa 500 der insgesamt 8400 landesweit geäußerten ernstlichen Sterbehilfeersuchen von Personen stammten, die „mit dem Leben abgeschlossen" hatten, jedoch nicht an einer ernsthaften Erkrankung litten. Die Studie kommt zwar zu dem Ergebnis, dass derartigen Ersuchen durch die befassten Ärzte nur äußerst selten Folge geleistet wurde, konstatiert jedoch auch, dass immerhin $28 \%$ der befragten Ärzte Beihilfe zum Selbstmord bei lebensüberdrüssigen Patienten jedenfalls in Ausnahmefällen für vertretbar halten. Der letztgenannte Befund dürfte eine gesteigerte Verantwortung der organisierten niederländischen Ärzteschaft für die Erarbeitung einer konsistenten Position zu dem hier erörterten, intrikaten Problem begründen. Der zu besprechenden Publikation ist zu diesem Zweck eine profunde Übersicht der für- und widerstreitenden Argumente zu entnehmen.

\section{Medizinrecht heute: Erfahrungen, Analysen, Entwicklungen (Festschrift 10 Jahre Arbeitsgemein- schaft Medizinrecht im DAV).}

Herausgegeben von der Arbeitsgemeinschaft Medizinrecht im DAV. Deutscher AnwaltVerlag, Bonn 2008, XXVI u. 990 S. geb., für Mitglieder der Arbeitsgemeinschaft kostenfrei

In 10 Jahren hat die „Arbeitsgemeinschaft Medizinrecht“ (Arge MedR) Großes geleistet: Tagungen, Fortbildungsveranstaltungen zur Gesundheitsreform, Arbeitsgruppen, Mitteilungsblatt, ZMGR Newsletter, Internetpräsenz, Wegbereiter des „Fachanwalt für Medizinrecht", Ausschuss für Medizinrecht und nun auch eine umfangreiche Festschrift: handlich, praktisch, informativ!

Auf knapp 1.000 Seiten sind nach einer Einführung zur Geschichte der Arge MedR (Freytag) 60 Beiträge abgedruckt zu den Bereichen: Recht der medizinischen Behandlung, Haftungsrecht, Vertragsarztrecht, Krankenhausrecht, Vertragsrecht, Berufsrecht, Strafrecht und Arzneimittel-, Medizinprodukte- und Apothekenrecht. Ein breites Spektrum, das die Sammelüberschrift „Medizinrecht heute“ auch deswegen verdient, weil - trotz Unterschieden im Einzelnen - alle Beiträge aktuell sind, die jeweilige Gesetzgebung (insbesondere VÄndG, GKV-WSG, z. T. auch GKV-OrgWG) und auch die Rechtsprechung berücksichtigen. 